\title{
SOLUTION OF TWO CONJECTURES IN SYMBOLIC DYNAMICS
}

\author{
K. H. KIM AND F. W. ROUSH
}

(Communicated by Thomas $\mathrm{H}$. Brylawski)

\begin{abstract}
We give an example of an inert involution on a subshift of entropy less than $\log 2$ which interchanges two fixed points. This proves simple finite order generation (FOG) of Wagoner [W2] is false and answers Questions 1.10 and 1.11 [W2]. We also give an example of two near Markov subshifts which are eventually conjugate but not conjugate, and a near Markov subshift with domain a full shift not equivalent to its time reversal. The former shows sofic shift equivalence does not coincide with strong sofic shift equivalence.
\end{abstract}

\section{INTRODUCTION}

One of the major problems in symbolic dynamics is FOG, finite order generation: the automorphism group of a full (Bernoulli) shift $S_{n}$ is generated by elements of finite order and shifts of $S_{t}, t \mid n$. This was conjectured by Frank Rhodes for $n=2$ in correspondence to Hedlund. A group of researchers centered at Berkeley and MSRI in 1983-1984 generalized it to automorphisms of subshifts of finite type which are inert, inducing the identity automorphism of the dimension group. Wagoner [W2] posed as a problem, which he called strong form of FOG, are inert automorphisms generated by simple involutions. Here we show the latter is false.

Many positive results have been obtained on FOG recently, the factorization of all 1-sided automorphisms in [BFK], the eventual factorization result in [W2] and [B2], and a density result in [KR3]. Ashley [A] has found an explicit set of generators for 1-sided automorphisms.

Here as usual a full $n$ shift $S_{n}$ is

$$
\stackrel{\infty}{i=-\infty}_{\infty}^{\infty}\{1,2, \ldots, n\}
$$

with product of discrete topologies. The shift map $\sigma(x)=y$ where $y_{i}=x_{i+1}$. A subshift $S_{A}$ of finite type associated with a 0-1 matrix $A=\left(a_{i j}\right)$ is the closed subset of all $x$ such that for all $i, a_{x_{i} x_{i+1}}=1$.

Received by the editors May 18, 1989 and, in revised form, December 3, 1989.

1980 Mathematics Subject Classification (1985 Revision). Primary 54H20; Secondary 28D20.

Key words and phrases. Finite order generation, inert automorphism, fixed point, orbit sign, time reversal, sofic strong shift equivalence, near Markov shift.

This work was partially supported by NSF DMS-8521533, 8820801 . 
A conjugacy is a homeomorphism commuting with the shift. An automorpirism is a self-conjugacy. Abstractly, the dimension group can be considered as the direct limit of the system

$$
\mathbf{Z}_{n} \stackrel{A}{\rightarrow} \mathbf{Z}_{n} \stackrel{A}{\rightarrow} \mathbf{Z}_{n} \stackrel{A}{\rightarrow} \cdots
$$

where $\mathbb{Z}_{n}$ denotes all vectors with integer coordinates. It also has a topological definition due to Krieger (see $\left.{ }^{3} B L R\right]$ ) which gives rise to an action of the automorphism group on it.

We may also associate a subshift of finite type to a matrix over $\mathbf{Z}^{+}$. To do this we label the edges in its graph and take all bi-infinite edge sequences in the graph. A simple automorphism is one which is conjugate to an automorphism permuting two edges between the same pair of vertices. Boyle [B1] has shown most known infinite classes of automorphisms are compositions of simple automorphisms.

\section{A NONSIMPLE INERT INVOLUTION}

Thie automorphism we give was found on the Cray X-MP/24 Alabama supercomputer.

The program runs through matrices $A$ of block form $\left[\begin{array}{ll}C & D \\ D & C\end{array}\right]$ where $C$ is $8 \times 8, A$ has involution switching $i$ and $i+8, i=1,2, \ldots, 8$. Here $C$ is fixed to have $c_{11}=1, c_{i, i+1}=1$, else $c_{i j}=0$. This seems a simple way to have two fixed points. $D$ ranges over partial permutations with two undefined values. For each matrix, it checks whether the first row block is identically equal to the second for $A^{16}$. By row sums, the entropy is small.

A second program simply computed $A, i=1$ to 16 , its trace and characteristic polynomial, and printed $A^{16}$ for a check.

Proposition 1. There exists an involution $\alpha$ of a subshift on sixteen symbols which interchanges two fixed points, is inert, and the subshift has entropy less than $\log 2$.

Proof. The subshift will be defined by a $16 \times 160-1$ matrix A. The matrix A has two cycles $\left(\begin{array}{llll}1 & 2 & \cdots & 8\end{array}\right)(910 \cdots 16)$, two loops $(1,1),(9,9)$, and elements $(i, m 1(i)+8),(i+8, m 1(i))$ where $m 1$ is the partial permutation, in which 0 denotes undefined,

$$
\left(\begin{array}{llllllll}
1 & 2 & 3 & 4 & 5 & 6 & 7 & 8 \\
0 & 2 & 8 & 3 & 6 & 4 & 5 & 0
\end{array}\right) .
$$

By construction $A P=P A$ where $P$ is the involution $\prod_{i=1}^{8}(i(8+i))$. Then $P$ is an involution giving an automorphism of the subshift reversing the fixed points corresponding to 1,9 . The entropy is the logarithm of the maximum eigenvalue. For it to be less than 2 it suffices that all row sums be less than or equal to 2, some row sum be less than 2, and the matrix be irreducible. (If all row sums equal 2 , the maximum eigenvalue is 2 , and if one is then reduced, this must reduce it, for an indecomposable matrix.) 
The action on the dimension group is induced by multiplication by $P$, once we take direct limits by $A$. For it to be trivial it suffices that $A^{16} P=A^{16}$.

We remark that the above proposition can be verified by computer.

Wagoner [W1, W2] has defined a space $R S(\bar{E})$ based on strong shift equivalences over $\mathbf{Z}^{+}$and a space $S(\bar{E})$ based on shift equivalences over $\mathbf{Z}^{+}$. Vertices are matrices, edges are (strong) shift equivalences, triangles are homotopies of (strong) shift equivalences. For complete details, see [W1] and [W2].

By $H_{1}$ we mean the first homology group. The first homology group is its abelianization.

Theorem 2. (i) The involution $\alpha$ is not a composition of simple involutions.

(2) The map $H_{1}(R S(\bar{E})) \rightarrow H_{1}(S(\bar{E}))$ has nonzero kernel.

(3) The orbit permiutation signs of involution $\alpha$ do not lie in the image of the subgroup generated by simple involutions.

Proof. By [B1] any simple involution fixes period 1 orbits if the entropy is less than or equal to 2 and the shift is not conjugate to a 2 -shift. This proves (1) and (3).

The homology of $R S(\bar{E})$ in dimension 1 is by [W1] the homology of the automorphism group modulo simple involutions. The map to the homology of $S(\bar{E})$ gives the action on dimension groups [W1]. Then $\alpha$ is nonzero in $H_{1}(R S(\bar{E}))$ detected by orbit signs but maps to zero in $H_{1}(S(\bar{E}))$. The first homology group of a group is isomorphic to its abelianization. This proves (2).

Proposition 3. The subshift $S_{A}$ is shift equivalent to $S_{B}$ where $B$ is the quotient of the graph $A$ by the $\mathbf{Z}_{2}$ action

$$
B=\left[\begin{array}{ccccccccc}
1 & 1 & 0 & 0 & \mid & 0 & 0 & 0 & 0 \\
0 & 1 & 1 & 0 & 0 & 0 & 0 & 0 & 0 \\
0 & 0 & 0 & 1 & \mid & 0 & 0 & 0 & 1 \\
0 & 0 & \frac{1}{0} & 0 & \mid & \frac{1}{1} & 0 & 0 & 0 \\
0 & 0 & 0 & 0 & 0 & \frac{0}{2} & 0 & 0 \\
0 & 0 & 0 & 1 & 0 & 0 & 1 & 0 \\
0 & 0 & 0 & 0 & 1 & 1 & 0 & 0 & 1 \\
1 & 0 & 0 & 0 & 0 & 0 & 0 & 0
\end{array}\right] .
$$

Proof. Write $A$ as

$$
\left[\begin{array}{c:c}
C & D \\
\hdashline D & C
\end{array}\right]
$$

and $B$ as $[C+D]$. Then a shift equivalence is given by $R=[I I]$,

$$
S=\left[\begin{array}{l}
\frac{(C+D)^{16}}{2} \\
\frac{(C+D)^{16}}{2}
\end{array}\right] .
$$

Since $A^{15}$ has all four blocks equal, the relation $R A^{16}=B^{16} R$ gives their value as $(C+D)^{16} / 2$. 
Note that the matrix $B$ is nonsingular and its characteristic polynomial is $x^{8}-2 x^{7}-2 x^{5}+6 x^{4}-2 x^{3}-4 x^{2}+6 x-2$.

Now we give solution to a couple of problems raised by Wagoner in [W2] based on the above results. Let $\pi_{2}$ denote the second homotopy group of a simplicial complex.

Question 1.10 of [W2] states, "Is $\pi_{2}(S(\bar{E}), R S(\bar{E}), A)=0$ ?" The answer is no since, as Wagoner writes, it would imply every element of the kernel of the dimension group representation is a product of simple elements.

Let $\mathrm{Aut}_{0}$ denote the group of inert automorphisms.

Question 1.11 of [W2] is "Is every finite order element of $\mathrm{Aut}_{0}\left(\sigma_{A}\right)$ simple?" Since our example has order 2 , the answer is no.

\section{Sofic Williams conjecture}

Perhaps the best-known unsolved problem in symbolic dynamics is the Williams conjecture.

Definition. Two matrices A, B over a semiring are shift equivalent if and only if there exist matrices $\mathrm{R}, \mathrm{S}$ over the semiring and $n \in \mathbf{Z}^{+}$with $R A=B R$, $A S=S B, R S=B^{n}, S R=A^{n}$. Strong shift equivalence is the transitive closure of this relation restricted to $n=1$. Here $R, S$ can be rectangular matrices.

Williams, in the landmark paper [Wi], showed that strong shift equivalence over $\mathbf{Z}^{+}$is equivalent to topological conjugacy and that it implies shift equivalence, for finite-type subshifts. At first he mistakenly stated the converse also (see [Wi]). Since then the converse has been known as the Williams conjecture but has never been proved. Because of [KR1] it would imply a decision procedure for topological conjugacy.

Nasu $[\mathrm{N}]$ and Nasu and Hamachi $[\mathrm{HN}]$ extended the Williams classification theorem to sofic subshifts, images of finite-type subshifts in full shifts under shift homomorphisms. But now one should use matrices in noncommuting variables, or a semigroup semiring. Boyle and Krieger [BK] defined a corresponding shift equivalence relation and showed it was equivalent to eventually sofic conjugacy, that is, conjugacy of all large powers of sofic subshifts. Kim and Roush [KR2] showed this relation was decidable.

Here we show as a consequence of work of Boyle and Krieger [BK] and Kim and Roush [KR3] that the extension to sofic shifts of the Williams conjecture is false.

Theorem 3.1. There exist near-Markov subshifts obtained by identifying all period 6 orbits in a 2-shift to a single period 6 orbit, which are eventually conjugate but not conjugate. There exists a near-Markov subshift of this type not equivalent to its time reversal.

Proof. Let $S_{A}$ be a 2-shift. By Theorem 3.9 of [KR3] and the example following, all order 6 gyration numbers are congruent to 0 modulo 3 . Let $S_{\theta}$ be the 
subshift of all period 6 points. By Theorem 3.2 of [BK], sofic quotients whose multiplicity set lies in $S_{\theta}$ are classified by (i) a quotient map $f$ on $S_{\theta}$ and (ii) a double coset of an automorphism $U$ of $S_{\theta}$. The two groups involved are (i) the group $Q_{f}$ of automorphisms of $S_{\theta}$ compatible with $f$ and (ii) the group of restrictions of automorphisms of $S_{A}$. Let $f$ identify all order 6 orbits to a single orbit. Then the maps compatible with $f$ factor as those interchanging orbits keeping positions within orbits fixed composed with those advancing each orbit the same amount. Either way in $Q_{f}$ order 6 gyration numbers are zero. Also in the restrictions modulo 3 gyration numbers are zero. So the modulo 3 gyration number is a double coset invariant. If we let $U$ move only one orbit then we can obtain zero or nonzero values. So there are nonconjugate sofic subshifts. But by Theorem 3.5 of [BK] they are eventually conjugate.

By Theorem 4.4 of [BK] any time reversal on the full shift would send a double coset $Q_{f} U(\text { Aut } S)_{\theta}$ to a double coset $Q_{f} W V^{-1} U V(\text { Aut } S)_{\theta}$.

The time reversal $V$ will send $U$ to its inverse up to conjugacy and composition with 2-shift automorphism. The class of $W$ depends only on the fixed time reversal of the 2-shift and on $f$. So if $x$ is the gyration number modulo 3 of the original double coset, its image has the form $w-x(\bmod 3)$. For suitable $x$, the two must not be equal, else $2 x \equiv w(\bmod 3)$ identically.

\section{Conclusion}

More recently, Boyle and Fiebig have provided further analysis of finite order inert automorphisms giving necessary and sufficient conditions for existence with specified action on periodic points.

\section{ACKNOWLEDGMENT}

The authors would like to express sincere thanks to Mike Boyle, Doug Lind, Bruce Kitchens, and the referee for very constructive comments.

\section{REFERENCES}

[A] J. Ashley, Marker automorphisms of the one sided d-shift, Ergodic Theory and Dynamical Systems (to appear).

[B1] M. Boyle, Nasu's simple automorphisms, Proc. Dynamical Systems (Univ. of Maryland, 1986-1987) (J. Alexander, ed.), Lecture Notes in Math., no. 1342, Springer-Verlag, Berlin, 1988.

[B2] _ Eventual extensions of finite codes, Proc. Amer. Math. Soc. 104 (1988), 965-972.

[BK] M. Boyle and W. Krieger, Almost Markov and shift equivalent sofic systems, Proc. of Dynamical Systems (Univ. of Maryland, 1986-1987) (J. Alexander, ed.), Lecture Notes in Math., vol. 1342, Springer-Verlag, Berlin, 1988.

[BFK] M. Boyle, D. Lind, and B. Kitchens, Automorphisms of one-sided subshifts of finite type, Ergodic Theory and Dynamical Systems 10 (1990), 421-449.

[BLR] M. Boyle, J. Franks, and D. Rudolph, On the automorphism group of a subshift of finite type, Trans. Amer. Math. Soc. 306 (1988), 71-114. 
[KR1] K. H. Kim and F. W. Roush, Decidability of shift equivalence, Proc. of Dynamical Systems (Univ. of Maryland, 1986-1987) (J. Alexander, ed.), Lecture Notes in Math., vol. 1342, Springer-Verlag, Berlin, 1988.

[KR2] _ An algorithm for sofic shift equivalence, Ergodic Theory and Dynamical Systems 10 (1990), 381-393.

[KR3] _ Structure of inert automorphisms of subshifts, Trans. Amer. Math. Soc. (submitted).

[W1] J. Wagoner, Triangle identities and symmetries of a subshift of finite type, Pacific J. Math. (to appear).

[W2] _ Eventual finite order generation for the kernel dimension group representation, Trans. Amer. Math. Soc. 317 (1990), 331-350.

[Wi] R. F. Williams, Classification of shifts of finite type, Ann. of Math. $\$ 8$ (1973), 120-153; errata, Ann. of Math. 99 (1974), 380-381.

Mathematics Research Group, Alabama State University, Montgomery, Alabama 36101-0271 